\title{
Linking of Items in Two Function-related Questionnaires to the International Classification of Functioning, Disability and Health: Shoulder Pain
}

\author{
Hae Jung Lee, Ju Min Song \\ Department of Physical Therapy, College of Health and Welfare, Silla University, Busan, Korea
}

\begin{abstract}
Purpose: This study was to identify international classification of functioning, disability and health (ICF) categories that could be linked conceptually to disability of arm, shoulder and hand (DASH) items and short form of health survey 36 (SF-36) items for persons with shoulder pain.

Methods: Linkage between each item in DASH and SF-36 and the categories in the ICF were assessed. The linking process was performed by ten health professionals following the linking rule. One hundred four patients with shoulder pain were enrolled from 12 private clinic outpatient departments and participated in this study. Pearson correlation coefficients were used to assess the relationships between each scale item and the linked ICF code.

Results: Thirty DASH items were able to be linked to 30 ICF codes, whereas the 36 items in SF-36 were only linked to 17 ICF codes. General health items included in SF-36 could not be linked to a relevant ICF concept. There was a high correlation between the two measurement tools and the linked ICF codes, DASH and its ICF code list $(r=0.91)$, SF-36-Physical Health and its code list $(r=-0.62)$, and SF36-Mental Health and its code list $(r=-0.72)$.

Conclusion: The results suggest that concepts within each item in DASH can be linked to ICF codes for patients with shoulder pain, however, the concepts in the SF-36 items had limited linkage to ICF codes. The shoulder-specific functional tool, DASH can be expressed with ICF codes and, therefore, its use can promote data standardization and improve communication between professionals.
\end{abstract}

Keywords: ICF, Shoulder pain, Functional measurement, DASH, SF-36

\section{INTRODUCTION}

Shoulder pain is one of the most common musculoskeletal conditions in outpatient settings. The number of patients who obtain treatment for shoulder pain has been increasing, and in 2016, it was reported that 110,000 patients with shoulder pain visited health professionals in Korea, 9th most sought-after treatment in Korea. ${ }^{1}$ Persons with shoulder pain can spend much effort on obtaining substantial medical service and can experience time loss from work as well as disability. Shoulder pain can contribute to individual suffering and be an economic burden for society. ${ }^{2}$

Shoulder pain may be the result of degenerative changes in joints, sports injuries, over use syndrome or muscle fatigue. ${ }^{2}$ Persons with

Received Nov 20, 2018 Revised Dec 28, 2018

Accepted Dec 31, 2018

Corresponding author Ju Min Song

E-mail jmsong@silla.ac.kr shoulder pain often experience limitations or restrictions of upper limb movements in daily activities, as well as during work and leisure activities. Sometimes persons with shoulder pain complain about sleep disturbance and psychological burdens including depression and stress. ${ }^{3}$

Functional parameter measurement is commonly used to understand patients' status of health and disability in rehabilitation medicine. ${ }^{4} \mathrm{~A}$ functional data instrument is designed to assess the effects of health conditions on the performance of everyday functions and is widely used by clinicians and clinical researchers. ${ }^{5,6}$ The disability of arm, shoulder and hand (DASH) instrument was developed to measure pain and disability associated with single or multiple disorders in the upper limb, and it is one of most widely used tools in

Copylight (C2018 The Korea Society of Physical Therapy

This is an Open Access article distribute under the terms of the Creative Commons Attribution Non-commercial License (Http:// creativecommons.org/license/by-nc/4.o.) which permits unrestricted non-commercial use, distribution, and reproduction in any medium, provided the original work is properly cited. 
clinical settings. DASH is reported to include items related to physical functions, symptoms and social roles. ${ }^{7}$ Short form of health survey 36 (SF-36) is a generic measurement tool and one of the most commonly used instruments for assessing health-related quality of life. It is considered useful for health status comparisons between patients with the same condition or between patients with different conditions. ${ }^{8}$ It has been reported that both tools, DASH and SF-36 could be administered in general populations. ${ }^{9,10}$ The international classification of functioning disability and health (ICF) contains items from biopsychosocial perspectives and is a common framework and classification for content analysis of functional measurements. ${ }^{11}$

The items in each instrument include concepts of what is meant to be measured and the instrument's total score can be used to describe and summarize person's health status or level of functioning. There are many instruments measuring health conditions and functional status. Those instruments have items that contain concepts that are similar among instruments, however, the ranges of frequency or intensity within those items may not be similar across all tools. Therefore, it may difficult to compare the results obtained by using different instruments. Using a common framework is useful to when comparing results from different measurement tools with similar concepts, and such a framework can be helpful when sharing information or communicating between health professionals about a patient's condition. ${ }^{12}$

The purposes of the study were to link conceptually the items included in the DASH and SF-36 instruments to the appropriate ICF categories and to examine the relationships between those measurement instruments and the linked ICF items using data obtained from subjects with shoulder pain.

\section{METHODS}

\section{Subjects}

\section{1) Subjects involved linking process}

Ten health professionals were invited to produce linked ICF code lists for the DASH and SF-36 items. Health professionals included five physiotherapists, one occupational therapist, one rehabilitation medical doctor, one orthopedic doctor, and two clinical researchers. All participants had ICF knowledge and experience in using clinical measurement tools.

\section{2) Clinical subjects}

Volunteers were recruited from 12 outpatient department of physiotherapy clinics. To be included in the study, participants needed to be over 18 years of age, and have shoulder pain. Volunteers were excluded from participation if they had a medical condition that produces shoulder symptoms such as breast cancer, fractures, or lymphedema. The study protocol was approved by human ethics committee of Silla University. All participants for the study provided informed consent prior to data collection.

\section{Experimental methods}

1) Study procedure

\section{(1) Linking process}

The ICF linking rule, proposed by Cieza and colleagues, ${ }^{13}$ was applied to develop a linked ICF code list for each clinical instrument. The process of linking between items of measurement and ICF categories was performed as follows: first, the concept were extracted from each item in each measurement tool (DASH and SF-36), the extracted concepts from each DASH and SF-36 item were evaluated and compared to ICF categories that contain the same concept; a linked ICF code list containing the conceptually similar ICF categories was made for each measurement instrument.

Linking between measurement tools and ICF categories was carried out by ten health professionals. Each participating professional independently created a list of linked ICF codes of each of the instrument tools. Each professional reported their linking results, then, results were compared and any discrepancies were discussed. All variations were integrated and a consensus was developed for clinical data collection. ICF code selection criteria did not limit the classification level when the concepts were consistent.

\section{(2) Clinical data collection}

To see if the list of linked ICF codes and each measurement tool item were related, clinical data were collected using DASH, SF-36 and each of the linked ICF code lists for those measurement tools.

Data were collected from patients with shoulder pain at twelve health institutes including a general hospital, private clinics located in Busan, Changwon, Daegu, Daejeon, Gwangju, Ulsan, and Jeju. Physiotherapists who had more than three years of clinical experience were involved in data collection. 


\section{2) ICF and measurement tools}

\section{(1) ICF and linked ICF codes}

ICF is one of international classifications developed by the World Health Organization to provide a standard language and framework for describing health and health-related states. It contains four components, body functions and structures, activities and participation, environmental factors and personal factors. The ICF comprises approximately 1,500 codes. ${ }^{11}$ As it is an international standard classification system, it can be used in various fields of study such as education, social welfare, policy making, and healthcare. The WHO conducts ICF updates annually, and the present study was based on the ICF 2017 version. ${ }^{14}$

Linked ICF codes for DASH and SF-36 and lists of the conceptually linked ICF categories for each tool were developed separately. The qualifier for the linked ICF items ranged from 0 (no difficulty) to 4 (extreme difficulty), with a higher score representing greater difficulty or disability.

\section{(2) DASH}

The DASH questionnaire is composed of 30 items measuring a person's ability to perform certain upper-extremity activities. It is a selfreport questionnaire that rates difficulties in daily activities on a 5-point Likert scale. Higher scores indicate a greater level of disability. The total test scores ranged from 0 (no disability) to 100 (most severe disability). It has been reported that DASH is highly reliable and valid to use in clinical settings for subjects with upper limb pain. ${ }^{7}$ Optional DASH modules (work, sports and performing arts modules) were not included in this study.

\section{(3) SF-36}

The SF-36 questionnaire is comprised of eight domains including physical functioning, role physical health, pain, general health, role emotional problem, emotional wellbeing, energy fatigue and social functioning. It is one of the most widely used measurement instruments among health care professionals. Higher scores represent a higher quality of life. It has been reported to have high reliability and validity.

\section{Data Analysis}

The general characteristics and measurement results of the subjects were analyzed by descriptive statistics. To examine associations between a list of linked ICF code and measurement tools, Pearson's correlation coefficients were calculated. All analyses in the study were performed using IBM SPSS 24 version.

\section{RESULTS}

\section{Characteristics of subjects}

One hundred four subjects (male: 60) with shoulder pain were included in the current study. Mean age was $44.67(S D \pm 15.60)$ years. Fifty-five participants reported right shoulder pain and 24 and 25 complained of pain in left shoulder or both shoulders, respectively. Mean and standard deviation of each measurement outcomes are reported in Table 1.

\section{Linking items of DASH, SF-36 into ICF categories}

The 30 DASH items were linked to 30 ICF categories. Nine catego-

Table 1. Means and standard deviations of DASH and SF36 measurement tools and of the linked ICF codes. SF36 is presented for both the physical health $(\mathrm{PH})$ and mental health $(\mathrm{MH})$ domains, and specific scales within each SF36 domain are also presented.

\begin{tabular}{|c|c|c|c|}
\hline Measurement tools & Mean (SD) & Linked ICF codes & Mean (SD) \\
\hline DASH & $39.95(22.65)$ & ICF-DASH & 36.39 (22.49) \\
\hline SF36-PH & $41.14(13.28)$ & ICF-SF36-PH & $13.93(9.10)$ \\
\hline Physical Functioning & $35.19(24.13)$ & & \\
\hline Role physical health & $30.29(37.31)$ & & \\
\hline Pain & $48.15(26.61)$ & & \\
\hline General health & $50.91(17.33)$ & & \\
\hline SF36-MH & $48.55(24.51)$ & ICF-SF36-MH & $7.36(5.46)$ \\
\hline Role emotional problem & $36.86(45.01)$ & & \\
\hline Emotional wellbeing & $55.50(21.43)$ & & \\
\hline Energy fatigue & $44.76(21.50)$ & & \\
\hline Social functioning & 57.09 (29.19) & & \\
\hline
\end{tabular}


Table 2. Linking of DASH and SF-36 items into ICF categories. Concepts within each item in DASH and SF-36 were compared to the concepts in ICF codes in order to link them together.

\begin{tabular}{|c|c|c|c|}
\hline DASH (item number) & SF-36 (item number) & Concept & ICF category \\
\hline \multirow[t]{2}{*}{ • Confidence, self-efficacy (30) } & & - Confidence & • b1266 Confidence \\
\hline & $\begin{array}{l}\text { - Pep, energy, fatigue, } \\
\text { tired }(23,27,29,31)\end{array}$ & - Energy, fatigue & • b130 Energy and driving function \\
\hline \multirow[t]{3}{*}{ • Pain, sleep (29) } & & - Pain, sleep & $\begin{array}{l}\text { • b134 Sleep functions, } \\
\text { b28014 Pain in upper limb, } \\
\text { b28016 Pain in joints }\end{array}$ \\
\hline & • Careful (19) & - Attention & - b140 Attention function \\
\hline & $\begin{array}{l}\text { - Anxious, nervous, sad, calm, } \\
\text { peaceful, happy, downhearted } \\
(17-19,20,24-26,28,30,32)\end{array}$ & - Emotion & • b152 Emotional function \\
\hline \multirow[t]{2}{*}{ • Tingling sensation in an upper limb (26) } & & - Sensation in an upper limb & - b265 Touch function \\
\hline & - Pain $(21,22)$ & - Pain & • b280 Sensation of pain \\
\hline $\begin{array}{l}\text { - Pain in an upper limb (24), } \\
\text { pain during an upper limb activity (25) }\end{array}$ & & - Pain in an upper limb & $\begin{array}{l}\text { - b28014 Pain in upper limb, } \\
\text { b28016 Pain in joints }\end{array}$ \\
\hline • Stiffness in an upper limb (28) & & - Stiffness in an upper limb & • b710 Mobility of joint functions \\
\hline - Weakness in an upper limb (27) & & - Weakness in an upper limb & - b7301 Power of muscles of one limb \\
\hline \multirow[t]{2}{*}{ - Do daily routine, shoulder problems, past week (23) } & - Daily activities (13-16, 17-19) & - Daily activities & - d230 Carrying out daily routine \\
\hline & • Bending, kneeling, stooping (8) & - Changing positions & - d410 Changing basic body position \\
\hline - Carry a light object (10), carry a heavy object (11) & - Lifting, carrying (3-5) & - Carrying an object & - d430 Lifting and carrying objects \\
\hline - Write (2), use a knife to cut food (16) & & - Manipulating & • d4402 Manipulating \\
\hline • Push a heavy door (5) & • Pushing a vacuum cleaner (4) & - Pushing & • d4451 Pushing \\
\hline - Lift and release an object above head (6) & & - Lifting an object above head & $\begin{array}{l}\text { - d430 Lifting and carrying objects, } \\
\text { d4452 Reaching }\end{array}$ \\
\hline \multirow[t]{3}{*}{$\begin{array}{l}\text { - Open a jar (1), turn a key (3), } \\
\text { exchange a lightbulb (12) }\end{array}$} & & $\begin{array}{l}\text { - Turning or twisting, } \\
\text { reaching and turning }\end{array}$ & $\begin{array}{l}\text { - d4453 Turning or twisting the hands } \\
\text { or arms }\end{array}$ \\
\hline & - Walking (9-11) & - Walking & • d450 Walking \\
\hline & - Climbing (6-7), running (3) & - Climbing, running & - d455 Moving around \\
\hline \multirow[t]{2}{*}{ - Drive or use public transportation (20) } & & - Using transportation & $\begin{array}{l}\text { - d470 Using transportation, d475 } \\
\text { driving }\end{array}$ \\
\hline & • Bathing (12) & - Washing & • d510 Washing \\
\hline • Wash one's back (14) & & - Washing a body part & - d5100 Washing body parts \\
\hline \multirow[t]{2}{*}{ • Wash or dry hair (13) } & & - Washing or drying a body part & $\begin{array}{l}\text { - d5100 Washing body parts, } \\
\text { d5102 Drying oneself }\end{array}$ \\
\hline & - Dressing (12) & - Dressing & • d540 Dressing \\
\hline • Put on a pullover (15) & & - Putting on & - d5400 Putting on clothes \\
\hline • Prepare a meal (4) & & - Prepare a meal & - d630 Preparing meals \\
\hline • Do housework (9) & • House work (22) & - Doing housework & • d640 Doing housework \\
\hline • Do housework, wash walls, wash floors (7) & & - Cleaning a house & • d6402 Cleaning living area \\
\hline • Garden or do yard work (8) & & - Gardening & $\begin{array}{l}\text { - d6505 Taking care of plants, } \\
\text { indoors and outdoors }\end{array}$ \\
\hline $\begin{array}{l}\text { - Do social activities with family and friends, } \\
\text { shoulder problem, past week (22) }\end{array}$ & - Social activity with others $(20,32)$ & $\begin{array}{l}\text { - Doing social activities with } \\
\text { family and friends }\end{array}$ & $\begin{array}{l}\text { - d750 Informal social relationships, } \\
\text { d760 Family relationships }\end{array}$ \\
\hline - Sexual activities (21) & & - Sexual activities & • d7702 Sexual relationships \\
\hline - Work, shoulder problem, past week (23) & • Work (13-16, 17-19) & - Work & • d850 Remunerative employment \\
\hline $\begin{array}{l}\text { - Recreational activities: little effort (17), } \\
\text { heavy impact (18), light impact (19) }\end{array}$ & - Strenuous sport, bowling (3-4) & - Recreational activities & • d920 Recreation and leisure \\
\hline
\end{tabular}

ries belong to the ICF body function component and 21 were linked to the activity and participation component. There were no body structure or environmental components linked with the DASH items. Only 17 ICF categories were linked to the items in SF-36: 4 categories in the body function component; and 13 categories from activity and participation components. Items in the general health domain in SF-36 were not able to be linked to an ICF category. Linked items in each tool are reported in Table 2. 

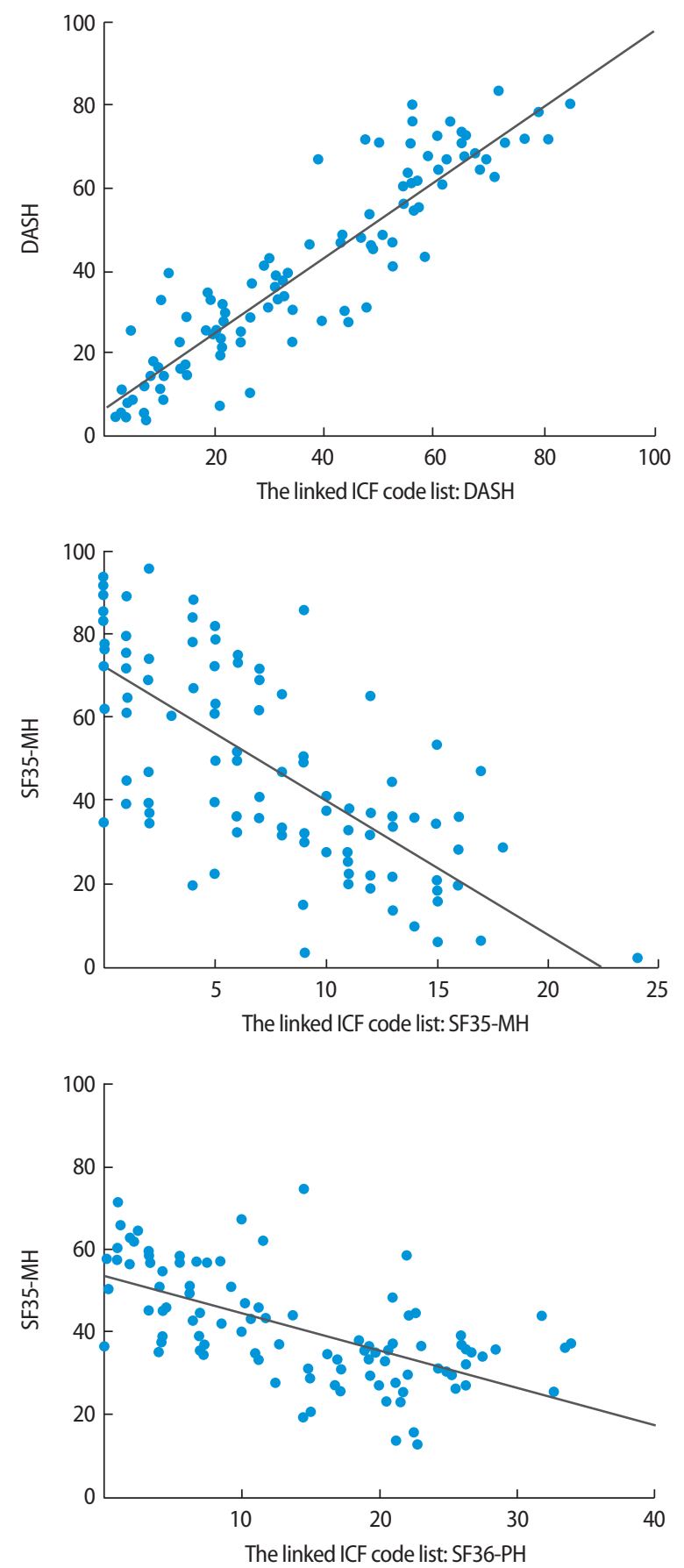

Figure 1. Correlation between the DASH, SF36-PH, and SF36-MH items and their linked ICF code lists.

\section{Associations between DASH and SF-36 and their linked ICF code lists}

Correlations between DASH and SF-36 and the instrument-specific linked code lists were assessed. The relationship between DASH and its linked ICF code list was very high $(\mathrm{r}=0.91, \mathrm{p}<0.01)$. SF-36 was divided into physical health and mental health domains. Rela- tionship between each domain and the SF-36 linked ICF code list were highly associated $(r=-0.62, p<0.01$ in physical health, $r=-0.72$, $\mathrm{p}<0.01$ in mental health) although the associations were negative, which means that higher scores in the linked ICF code list were associated with lower SF-36 scores (Figure 1).

\section{DISCUSSION}

This study was conducted to examine whether clinical measurement tool concepts could be linked to the ICF, which promotes data standardization and communication between professionals. DASH, which measures functional aspects of upper limb pain and disability, and SF-36, a questionnaire for assessing the quality of life, were chosen for conceptual-linking to ICF categories. Each of the items in those two instruments was linked to ICF code and lists of the linked ICF codes for each tool were developed. After examining clinical data from 104 patients with shoulder pain, it was determined that the relationships between the measurement tools and the relevant ICF code list were high.

The analyses showed that some of the items in the measurement tools contained various concepts, thus, they could be linked into more than one ICF codes. On the other hand, for some of items in measurement tools, one concept could be present in several items and those items were all linked to one ICF code.

Through the linking process, 30 items of DASH were linked to 30 ICF codes. The study results showed that symptom experience during a certain time period (e.g. 'during the past week' in DASH) could not be defined in ICF. In the current study, the concept of 'problem' could also not be specified to a certain concept or be linked to a specific ICF code. Another item in DASH describes pain related to performing any 'specific activity', and 'specific activity' could not linked to ICF. The term 'specific activity' may be commonly used in clinical settings when asking patients to explain their conditions.

In clinical measurement tools, it was often observed that one concept was present in several items with different ranges of intensity or frequency, and each of those items could be linked to one ICF code. ${ }^{15,16}$ This study showed that the level of performing certain activities was included in sequential items in both DASH and SF-36, such as carrying light object (item 10), carrying heavy object (item 11) in DASH, and climbing from one flight (item 6) to several flights 
of stairs (item 7) in SF-36. The concept related to executing the activity is the same in those items and, therefore, they could be linked to one ICF category (d430 lifting and carrying objects, d4551 climbing, respectively) whereas the intensity or extent of activities was not directly linked to ICF codes, as intensity of some activities is not included within ICF. These results were consistent with those in previous studies. ${ }^{13,17}$ However, walking distance from one block (item 11) to more than $1.5 \mathrm{~km}$ (item 9) were linked to $\mathrm{d} 4500$ walking short distances and d4501 walking long distances, respectively, in ICF.

Intensity is included in the concept of carrying out of various activities in both DASH and SF-36. This was detected in items 17 (card playing, knitting), 18 (hammering, golf), and 19 (Frisbee, badminton), which contained concept related to an impact force applied to the upper limb in DASH. SF-36 also has items 3 to 5 indicating various intensities or strength from light to vigorous activity. The concept of intensity in those items was not extractable, however, example activities within those items may reflect 'intensity' and could be linked to ICF codes.17 In clinical settings, intensity or extent of activities are useful when evaluating patient health and functional status, and is useful as an outcome measure for some treatments. ${ }^{5}$

Several items in DASH, such as item 1 'open a jar', item 3 'turn a key', and item 12 'exchange a light bulb', contain the same concept and each was linked to one ICF code (d4453 turning or twisting the hands or arms). Those items contain the same concept but each item in DASH describes a different wrist and hand activity; those results were consistent with those in previous studies. ${ }^{16,17}$

In a previous study into linking DASH and ICF, one item regarding self-efficacy and confidence was defined as a personal factor. ${ }^{17}$ Our result did not support that finding as self-efficacy and confidence in DASH was not considered to indicate a person's characteristics. Instead, it should be more closely related to biopsychological aspects and thus, it was linked to mental function (b1266 confidence) in a body function component affected by upper limb pain or problems. ${ }^{9}$

Stiffness in an upper limb (item 28) in DASH has been related to the sensation of muscle stiffness. ${ }^{17}$ However, we concluded that stiffness in an upper limb was associated with the concept of mobility of joint function because this item considered whether mobility limitation of the upper limb was present and there was interference with limb movement.

'Write' (item 2) and 'use of knife to cut food' (item 16) in DASH may be considered to be as a hand function rather than associated with the concept of applying knowledge or learning. A previous study reported that these items were linked to writing (d170) and eating (d550), respectively, in ICF.,17 However, 'write' and 'use of knife to cut food' may suggest fine hand use and manipulating (d4402) function in ICF. Manipulating is defined as "using fingers and hands to exert control over, direct or guide something, such as when handling coins or other small objects, such as scissors, shoe laces, pencils, chopsticks, knives and forks" and that was logically selected for linkage of the 'write' and 'use of knife to cut food' DASH items.

We found that some of the items in DASH and SF-36 include the same concept but to a different extent. This could indicate differences between specific and generic tools. ${ }^{15,16}$ Dressing (item 12) was included in SF-36, whereas putting on a pullover sweater (item 15) was in DASH, and those items were linked to dressing (d540) and putting on clothes (d5400), respectively. Other items, such as bathing (item 12) in SF-36 vs. washing one's back (item 14) and wash or dry hair (item 13) in DASH were linked to washing (d510), washing body parts (d5100), and drying oneself (d5102), respectively. They fall within the same concept, but they could be linked to different ICF codes due to different purposes or contents of the measurements. ${ }^{15,16}$ In DASH, some of the items include more than two concepts within a single item. For example, an item about lifting an object above the head (item 6) was suggested to include the concepts of lifting, carrying, and reaching, so, both lifting and carrying objects (d430) and reaching (d4452) in ICF were linked to those DASH items.

In conclusion, the study results suggest that the concepts in items in DASH, a shoulder-specific measurement tool, and SF-36, a generic tool for measuring the quality of life, can be linked to ICF, which is considered a common, standardized framework for health status and functioning. The results of our analyses may help to promote data standardization among measurement tools and help standardize communication between professionals. Further studies into linking data between ICF and various clinical measurement tools for shoulder pain and disability from various clinical settings are needed.

\section{REFERENCES}

1. Health Insurance Review \& Assessment Service. Health insurance statistics yearbook. 2017. http://www.hira.or.kr/main.do. 
2. Greenberg DL. Evaluation and treatment of shoulder pain. Med Clin. 2014;98(3):487-504.

3. Largacha M, Parsons IM, Campbell B et al. Deficits in shoulder function and general health associated with sixteen common shoulder diagnoses: a study of 2674 patients. J Shoulder Elbow Surg. 2006;15(1):30-9.

4. Lee HJ, Song JM. Which information is commonly used for patients with stroke at rehabilitation settings? J Kor Phys Ther. 2016;27(6):392-6.

5. Michener LA. Patient- and clinician-rated outcome measures for clinical decision making in rehabilitation. J Sport Rehabil 2011;20(1):37-45.

6. Lee $H$. Neck pain and functioning in daily activities associated with smartphone usage. J Kor Phys Ther. 2016;28(3):183-8.

7. Hudak PL, Amadio PC, Bombardier C. Development of an upper extremity outcome measure: the DASH (disabilities of the arm, shoulder and hand). Am J Ind Med. 1996;29(6):602-8.

8. Ware JE. SF-36 health survey update. Spine. 2000;25(24):3130-9.

9. Roe Y, Soberg HL, Bautz-Holter E et al. A systematic review of measures of shoulder pain and functioning using the international classification of functioning, disability and health (ICF). BMC Musculoskelet Disord. 2013;14(2):73.
10. Lins LE, Carvalho FM. Sf-36 total score as a single measure of health-related quality of life: scoping review. SAGE Open Med. 2016;4:1-12.

11. WHO. International classification of functioning, disability, and health: Korean version. Seoul, Ministry of Health and Welfare, 2016.

12. WHO. How to use the ICF: a practical manual for using the international classification of functioning disability and health (ICF): 0.9 version. Geneva, WHO, 2017.

13. Cieza A, Geyh S, Chatterii S et al. ICF linking rules: an update based on lessons learned. J Rehabil Med. 2005;37(4):212-8.

14. WHO. ICF. 2017. http://apps.who.int/classifications/icfbrowser/

15. Lee HJ, Song JM. Mapping items of functioning questionnaires into the international classification of functioning, disability and health: low back pain. J Kor Phys Ther. 2016;28(5):321-7.

16. Song JM, Lee HJ. Mapping items of functioning questionnaires into the international classification of functioning, disability and health: stroke. J Kor Phys Ther. 2016;28(5):341-7.

17. Drummond AS, Sampaio RF, Mancini MC et al. Linking the disabilities of arm, shoulder, and hand to the international classification of functioning, disability, and health. J Hand Ther. 2007;20(4):336-44. 